\title{
Beamstrahlung considerations in laser-plasma-accelerator-based linear colliders
}

\author{
C. B. Schroeder, E. Esarey, and W. P. Leemans \\ Lawrence Berkeley National Laboratory, Berkeley, California 94720, USA
}

(Received 23 November 2011; published 4 May 2012)

\begin{abstract}
Beam-beam interaction constraints modify the basic plasma density scalings for a linear collider based on laser-plasma accelerators. In the quantum beamstrahlung regime, it is shown that operating at low plasma density increases beamstrahlung effects, owing to the higher bunch charge and longer bunch length. At high plasma density, the bunch charge is limited by beam loading, and the required power is proportional to the square root of the plasma density. At low plasma density, the bunch charge is limited by beamstrahlung, which, for fixed luminosity, requires operation at higher laser repetition rate and, hence, higher power requirements, or the use of multibunch trains. If round beams are used in a multibunch train format with fixed beam loading, and the collider is constrained by beamstrahlung, then the required collider power is independent of plasma density.
\end{abstract}

DOI: 10.1103/PhysRevSTAB.15.051301

PACS numbers: 52.38.Kd, 41.75.Lx

\section{INTRODUCTION}

Advanced acceleration techniques are actively being pursued to expand the energy frontier of future colliders. It is anticipated that the next lepton collider will require $\gtrsim 1 \mathrm{TeV}$ center-of-mass energy [1]. This center-of-mass energy is near the limit of what can be constructed using conventional accelerator technology, given reasonable space and cost restrictions [2]. Laser-plasma accelerators (LPAs) have attracted significant interest as an accelerator technology because of their ability to sustain extremely large acceleration gradients, enabling compact accelerating structures [3]. Laser-plasma acceleration is realized by using a short-pulse, high-intensity laser to ponderomotively drive a large electron plasma wave (or wakefield) in an underdense plasma. The electron plasma wave has relativistic phase velocity, and can support large electric fields in the direction of propagation of the laser. Rapid progress in the field of laser-plasma acceleration, and in particular the demonstration of high-quality $\mathrm{GeV}$ electron beams using $\mathrm{cm}$-scale plasmas at Lawrence Berkeley National Laboratory [4], has increased interest in laserplasma acceleration as a path toward a compact, TeV-class, electron-positron linear collider $[5,6]$.

The basic plasma density $n$ and laser wavelength $\lambda$ scaling laws for a laser-plasma-based linac were presented in Ref. [6]. These scaling laws were derived under the assumptions of fixed final focusing to the interaction point (IP), fixed efficiency of the energy conversion, and a fixed center-of-mass energy and luminosity required for highenergy physics experiments. One of the conclusions of Ref. [6] was that the total required power for the collider

Published by the American Physical Society under the terms of the Creative Commons Attribution 3.0 License. Further distribution of this work must maintain attribution to the author(s) and the published article's title, journal citation, and DOI. scaled as $P_{\text {wall }} \propto \sqrt{n}$, where $n$ is the ambient plasma density. Recently, a low density operating regime for a laserplasma collider has been emphasized in Ref. [7] with a nominal operating density near $10^{15} \mathrm{~cm}^{-3}$. The reason for emphasizing this low density operating regime is that, as implied by the scaling laws derived in Ref. [6], operating at a density of $10^{15} \mathrm{~cm}^{-3}$ lowers the required collider power by an order of magnitude as compared to operating at a density of $10^{17} \mathrm{~cm}^{-3}$ (as well as lowering the accelerating field by an order of magnitude, and increasing the required laser pulse energy by 3 orders of magnitude). Although this scaling indicates lower densities are favorable for reduced power requirements, additional constraints may modify this basic scaling. In this work we examine the additional constraint, imposed by experimental high-energy physics, of fixed beamstrahlung-induced beam energy loss and photon emission. When operating at sufficiently low plasma density, the constraint of fixed beamstrahlung strongly modifies the basic plasma density scalings. In particular, the scalings for the required power imply that it is no longer advantageous to operate at low density $\left(\sim 10^{15} \mathrm{~cm}^{-3}\right)$ as opposed to more moderate densities (few $10^{16} \mathrm{~cm}^{-3}$ to a few $10^{17} \mathrm{~cm}^{-3}$ ).

Although many components of a LPA-based electronpositron collider, such as the injector and final focus design, are yet to be determined, scaling laws based on fundamental physical considerations may be derived. In this work, we show how beam-beam interaction constraints, that are strongly manifest when operating at low plasma density, modify the basic plasma density scalings and, hence, the LPA-collider design. We show that, for round beams at low plasma density, the bunch charge is limited by beamstrahlung effects. Furthermore, to maintain reasonable efficiency, one must consider multibunch operation in this beamstrahlung-limited regime. Using bunch trains and optimal beam loading, the required $\mathrm{AC}$ power is shown to be independent of plasma density and laser 
wavelength in the beamstrahlung-limited regime. We also discuss how laser technology may influence the optimal operating regime for an LPA-based collider.

\section{BEAMSTRAHLUNG CONSIDERATIONS}

The rate of events in a collider is determined by the product of the collision cross section and luminosity. The geometric luminosity is

$$
\mathcal{L}=\frac{f N^{2}}{4 \pi \sigma_{x}^{*} \sigma_{y}^{*}}=\frac{P_{b} N}{4 \pi U_{b} \sigma_{x}^{*} \sigma_{y}^{*}},
$$

where $f$ is the collision frequency, $N$ is the number of particles per bunch (here equal number of particles per bunch in the electron and positron beams is assumed), $\sigma_{x}^{*}$ and $\sigma_{y}^{*}$ are the horizontal and vertical rms beam sizes at the IP, $U_{\mathrm{cm}}=2 \gamma m_{e} c^{2}=2 U_{b}$ is the center-of-mass energy with $U_{b}$ the single beam energy, and $P_{b}=f N U_{b}$ is power in one beam. Since the cross section for collisions scales as the inverse of the square of the center-of-mass energy, $\propto U_{\mathrm{cm}}^{-2}$, the luminosity must increase proportionally to maintain the collision rate. The luminosity requirement is approximately $\mathcal{L}\left[10^{34} \mathrm{~cm}^{-2} \mathrm{~s}^{-1}\right] \approx U_{\mathrm{cm}}^{2}[\mathrm{TeV}]$. As the luminosity scaling indicates, for fixed beam power, the transverse beam density at the IP must be increased as the center-of-mass energy increases. Dense colliding beams may result in the focusing of one beam by the other, i.e., beam disruption [8]. Beam disruption is characterized by the disruption parameter $D_{x, y}=2 r_{e} N \sigma_{z} /\left[\gamma \sigma_{x, y}^{*}\left(\sigma_{x}^{*}+\sigma_{y}^{*}\right)\right]$, where $\sigma_{z}$ is the rms bunch length and $r_{e}=e^{2} / m_{e} c^{2}$ is the classical electron radius. Note that luminosity enhancement effects from beam disruption are typically weak $D<1$ for LPA-based colliders owing to the ultrashort LPA bunch lengths $\sigma_{z} \ll$ $\lambda_{p}$, where $\lambda_{p}=\left(\pi / r_{e} n\right)^{1 / 2}$ is the plasma wavelength, i.e., $\lambda_{p}[\mu \mathrm{m}] \simeq 3.3 \times 10^{10}\left(n\left[\mathrm{~cm}^{-3}\right]\right)^{-1 / 2}$.

There are many limitations to the achievable beam density at the IP. For example, some of these include limitations on the achievable beam emittance (given the initial emittance and cooling methods), radiation effects during the final focus to the IP, emittance growth in the linacs, and beam-beam interactions at the collision. In the following we consider how beam-beam interactions at the IP influence the operating parameters of a collider based on laser-plasma accelerators.

The beam-beam interaction at the IP produces radiation (beamstrahlung) that generates background photons for the detectors and increases the beam energy spread (resulting in loss of measurement precision) $[9,10]$. The beam-beam interaction is characterized by the Lorentz-invariant beamstrahlung parameter $Y$ (mean field strength in the beam rest frame normalized to the Schwinger critical field): $Y=\gamma\langle E+B\rangle / E_{c}$, where $E_{c}=m_{e}^{2} c^{3} / e \hbar$ is the Schwinger critical field. For a Gaussian beam, the average beamstrahlung parameter is [10]

$$
\Upsilon \approx \frac{5 r_{e}^{2} \gamma}{6 \alpha\left(\sigma_{x}^{*}+\sigma_{y}^{*}\right)} \frac{N}{\sigma_{z}},
$$

where $\alpha=e^{2} \hbar / c$ is the fine structure constant. As Eq. (2) indicates, using flat beams $\sigma_{x}^{*} / \sigma_{y}^{*} \ll 1$ reduces the beamstrahlung. Although, in principle, beams with highly asymmetric transverse emittances $\epsilon_{x} \ll \epsilon_{y}$ may be accelerated in LPAs using highly asymmetric transverse laser modes (to create asymmetric focusing forces), in the following we will consider round beams $\sigma_{x}^{*}=\sigma_{y}^{*}=\sigma_{*}$. In addition to removing the need for highly asymmetric laser modes, using round beams removes the need for damping rings to produce asymmetric emittances and greatly reduces the alignment tolerances at the final focus.

In terms of the beamstrahlung parameter (and assuming negligible disruption), the average number of emitted photons per electron is [10]

$$
n_{\gamma} \approx 2.54\left(\frac{\alpha^{2} \sigma_{z}}{r_{e} \gamma}\right) \frac{\Upsilon}{\left(1+\Upsilon^{2 / 3}\right)^{1 / 2}},
$$

which characterizes the background level in the detector. The average fractional energy loss from beamstrahlung is [10]

$$
\delta_{b} \approx 1.24\left(\frac{\alpha^{2} \sigma_{z}}{r_{e} \gamma}\right) \frac{\Upsilon^{2}}{\left[1+(3 \Upsilon / 2)^{2 / 3}\right]^{2}},
$$

which describes the broadening of the luminosity energy spectrum. Equations (3) and (4) assume a Gaussian bunch. For high-energy physics experiments it is desirable to minimize Eqs. (3) and (4). For example, the proposed $3 \mathrm{TeV}$ Compact Linear Collider (CLIC) design considers $n_{\gamma}=2.1$ with a beamstrahlung-induced relative energy loss of $28 \%$ [11].

The next-generation linear colliders with $U_{\mathrm{cm}} \gtrsim 1 \mathrm{TeV}$ will most likely operate in the quantum beamstrahlung regime with $Y \gg 1$. In the quantum beamstrahlung regime $Y \gg 1$, the average number of emitted photons per electron and the fractional beamstrahlung-induced energy loss are

$$
n_{\gamma} \simeq 2.54\left(\alpha^{2} / r_{e} \gamma\right) \sigma_{z} \Upsilon^{2 / 3} \text {, }
$$

and

$$
\delta_{b} \simeq 0.722\left(\alpha^{2} / r_{e} \gamma\right) \sigma_{z} \Upsilon^{2 / 3},
$$

respectively. Using Eq. (2), the number of beamstrahlung photons per electron and the average relative energy loss scale as [12]

$$
n_{\gamma} \simeq 3.5 \delta_{b} \propto \frac{N^{2 / 3} \sigma_{z}^{1 / 3}}{\sigma_{*}^{2 / 3} \gamma^{1 / 3}},
$$

in the regime $Y \gg 1$. As Eq. (7) indicates, beamstrahlung effects are reduced by using shorter bunches and smaller charge per bunch. Plasma-based accelerators are intrinsically sources of ultrashort bunches since the scale length of 
the accelerating bucket is the plasma wavelength. Reduction in the bunch length for fixed charge is limited by bunch density constraints, i.e., $n_{b} \lesssim n_{0}$ to avoid the blow-out regime [13] and the resulting strong beam selffocusing and emittance growth [14]. For a fixed luminosity goal, reduction in charge per bunch to control beamstrahlung must be accompanied with an increase in the repetition rate $f$ (such that $\mathcal{L}=$ constant), and, hence, an increase in the power requirements.

Without additional constraints (e.g., reducing beamstrahlung) the maximum charge that can be loaded into a plasma wave is given by the beam loading (BL) limit, $N \leq N_{\mathrm{BL}} \propto n^{-1 / 2}$. The beam loading limit is determined by the amount of charge required to excite a beam-driven wake that approximately cancels the laser-driven wave, thereby absorbing the plasma wave energy for high efficiency $[3,15]$. But, if we assume a fixed number of beamstrahlung photons per lepton produced at the IP (that is acceptable for a given collider detector design), then using Eq. (5) the charge per bunch must be sufficiently small such that

$$
N \leq N_{\text {beam }}=\frac{0.6 n_{\gamma}^{3 / 2}}{\alpha^{2} r_{e}^{1 / 2}} \frac{\gamma^{1 / 2} \sigma_{*}}{\sigma_{z}^{1 / 2}} .
$$

This beamstrahlung constraint is in addition to the beam loading constraint $N \leq N_{\mathrm{BL}}$. Therefore, for collider applications with a maximum acceptable beamstrahlung, the bunch charge must be less than the smaller of $N_{\text {beam }}$ and $N_{\text {BL }}$. Typically, for high plasma densities (e.g., $n \gtrsim$ $10^{17} \mathrm{~cm}^{-3}$ ) the bunch charge is limited by beam loading $N \leq N_{\text {BL }}<N_{\text {beam }}$, and for low plasma densities (e.g., $n \lesssim$ $10^{16} \mathrm{~cm}^{-3}$ ) the bunch charge will be limited by beamstrahlung considerations $N \leq N_{\text {beam }}<N_{\mathrm{BL}}$.

Using Eqs. (1) and (8), the luminosity per $U_{\mathrm{cm}}^{2}$ (i.e., the required luminosity for a given center-of-mass energy) scales as

$$
\frac{\mathcal{L}}{U_{\mathrm{cm}}^{2}} \propto \frac{n_{\gamma}^{3 / 2} \eta P_{\text {wall }}}{\sigma_{*} \gamma^{5 / 2} \sigma_{z}^{1 / 2}}=\frac{n_{\gamma}^{3 / 2} \eta P_{\text {wall }}}{\beta_{*}^{1 / 2} \epsilon_{n}^{1 / 2} \gamma^{2} \sigma_{z}^{1 / 2}},
$$

where $\eta=P_{b} / P_{\text {wall }}$ is the efficiency of energy transfer from the AC power source to the beam, $\epsilon_{n}$ is the normalized transverse emittance, and $\beta_{*}$ is the IP beta function. The beam size at the IP will be determined by the achievable emittance injected into the LPA linac and the final focus optics, $\sigma_{*} \gamma^{1 / 2}=\left(\beta_{*} \epsilon_{n}\right)^{1 / 2}$. Limitations on the final focus optics $\beta_{*}$, e.g., from synchrotron radiation [16], imply novel cooling methods at the injector are required for next-generation linear colliders to reduce the transverse emittance. Given fixed $\epsilon_{n}$ from the injector, fixed $\beta_{*}$ from the final focus optics, and fixed $n_{\gamma}$ from detector constraints, Eq. (9) indicates that short bunches will reduce the required beam power to achieve a luminosity goal [2]. As discussed below, in general, the bunch length will scale with the plasma wavelength $\sigma_{z} \propto \lambda_{p} \propto n^{-1 / 2}$, and hence, for fixed beamstrahlung, higher luminosity per beam power $\mathcal{L} / P_{b}$ is achieved by operating at higher plasma densities.

\section{PLASMA DENSITY SCALINGS}

The basic plasma density and laser wavelength scalings for an LPA accelerator stage were presented in Ref. [6]. In particular, the accelerating gradient scales as $E_{z} \propto n^{1 / 2}$, the energy gain per LPA stage scales as $U_{\text {stage }} \propto n^{-1}$, the laser energy required to power a single LPA stage scales as $U_{L} \propto$ $n^{-3 / 2} \lambda^{-2}$, the length of an LPA stage scales as $L_{\text {stage }} \propto$ $n^{-3 / 2} \lambda^{-2}$, and, for a required beam energy $U_{b}$, the number of LPA stages in the linac scales as $N_{\text {stage }} \propto n \lambda^{2}$. Table I shows the basic LPA plasma density and laser wavelength scalings, independent of limitations on the bunch charge. These scalings assume the normalized laser parameters $a_{0}$, $k_{p} c \tau_{L}, k_{p} r_{L}$ are held constant, where $a_{0}$ is the laser strength parameter, $\tau_{L}$ is the laser pulse duration, and $r_{L}$ is the laser spot size. With these scalings various operating points may be explored. For example, using these plasma density scalings [6], various sets of LPA-collider parameters were explored in Refs. [6,7]. In particular, Nakajima et al. [7] considered a low plasma density regime, but neglected to consider IP constraints (e.g., Ref. [7] assumed focused beam sizes at the IP several orders of magnitude below the Oide limit [16]) and neglected the limitations imposed by beamstrahlung at low plasma density, as discussed in this work.

If we consider fixed beam loading, i.e., energy transfer from the plasma wave to the beam given acceptable beam quality and accelerating gradient, then the efficiency of energy transfer from plasma wave to beam will be constant $\eta_{p b}=N U_{\text {stage }} / U_{L}=$ constant. Using the scalings above, constant beam loading efficiency using a single bunch implies $N \sim N_{\mathrm{BL}} \propto n^{-1 / 2}$. It should be emphasized that a collider will almost certainly use shaped bunches such that high efficiencies can be achieved without energy spread growth [15]. Also, to avoid dephasing limitations to the energy gain requires tapering the plasma [17]. Avoiding dephasing by tapering will greatly improve the efficiency

TABLE I. Laser-plasma accelerator plasma density and laser wavelength scalings [6].

\begin{tabular}{lc}
\hline \hline Parameter & Scaling \\
\hline Accelerating field, $E_{z}$ & $n^{1 / 2}$ \\
Length (single LPA stage), $L_{\text {stage }}$ & $n^{-3 / 2} \lambda^{-2}$ \\
Energy gain (single LPA stage), $U_{\text {stage }}$ & $n^{-1} \lambda^{-2}$ \\
Number of stages, $N_{\text {stage }}$ & $n \lambda^{2}$ \\
Total length, $L_{\text {total }}$ & $n^{-1 / 2}$ \\
Laser pulse duration, $\tau_{L}$ & $n^{-1 / 2}$ \\
Laser pulse spot size, $r_{L}$ & $n^{-1 / 2}$ \\
Peak laser power (single LPA stage), $P_{\text {peak }}$ & $n^{-1} \lambda^{-2}$ \\
Laser energy (single LPA stage), $U_{L}$ & $n^{-3 / 2} \lambda^{-2}$ \\
\hline \hline
\end{tabular}


of energy transfer from the laser to the beam $\eta_{L p} \eta_{p b}$ by increasing the effective laser-plasma interaction length (thereby increasing the efficiency of energy transfer from laser to plasma wave $\eta_{L p}$ ). Without tapering, the efficiency of the LPA in the quasilinear regime will be poor owing to dephasing [18].

Beam loading considerations indicate, for fixed loading of the plasma wave, that $\left(n_{b} / n_{0}\right) k_{p} \sigma_{z}=$ constant, where $n_{b}$ is the beam density, and $k_{p} \sigma_{r}=$ constant, where $\sigma_{r}$ is the beam radius in the accelerator. Hence, for fixed ratio of beam density to plasma density $n_{b} / n_{0}$, the bunch length scales as $\sigma_{z} \propto k_{p}^{-1} \propto n^{-1 / 2}$. In the present concept, the focusing forces acting on the beams originate from the transverse laser-driven wakefields in the plasma. It should be noted that the beam radius $\sigma_{r}$ can be controlled by controlling the transverse wakefields using shaped transverse laser intensity profiles in the quasilinear regime [19]. Independent control of the transverse focusing and longitudinal accelerating forces and, hence, the beam radius, in the LPA is critical for control of emittance [20]. With the plasma density scalings for the bunch charge and length set by beam loading, the average number of emitted photons per electron and the average relative energy loss Eq. (7) scale as

$$
n_{\gamma} \simeq 3.5 \delta_{b} \propto N^{2 / 3} \sigma_{z}^{1 / 3} \propto n^{-1 / 2},
$$

indicating reduced beamstrahlung effects at higher plasma density. Table II shows the plasma density and laser wavelength scalings [6] in the regime not limited by beamstrahlung at the IP, $N=N_{\mathrm{BL}}<N_{\text {beam. }}$.

\section{A. Beamstrahlung-limited regime}

If beamstrahlung effects are too severe (e.g., in the low plasma density regime), then one must consider operating with charge per bunch below the beam loading limit, satisfying Eq. (8). If the bunch length scales linearly with plasma wavelength $\sigma_{z} \propto \lambda_{p} \propto n^{-1 / 2}$, then

$$
N \simeq N_{\text {beam }} \propto \sigma_{z}^{-1 / 2} \propto n^{1 / 4} .
$$

If the amount of beamstrahlung that can be tolerated at the IP is constrained (e.g., $n_{\gamma} \simeq 2$ ), then the luminosity obtained per beam power expended, from Eq. (1), scales as $\mathcal{L} / P_{b} \propto n^{1 / 4}$, indicating higher plasma density (short

TABLE II. Plasma density and laser wavelength scalings in the regime not limited by beamstrahlung ( $N=N_{\text {BL }}<N_{\text {beam }}$ ) [6].

\begin{tabular}{lc}
\hline \hline Parameter & Scaling \\
\hline Bunch charge, $N$ & $n^{-1 / 2}$ \\
Bunch duration, $\sigma_{z}$ & $n^{-1 / 2}$ \\
Beamstrahlung photons per electron, $n_{\gamma}$ & $n^{-1 / 2}$ \\
Laser repetition rate, $f_{L}$ & $n$ \\
Average laser power, $P_{\text {avg }}$ & $n^{-1 / 2} \lambda^{-2}$ \\
Total AC power, $P_{\text {wall }}$ & $n^{1 / 2}$ \\
\hline \hline
\end{tabular}

beams) is preferable for fixed beamstrahlung background and beamstrahlung-induced energy spread. Operating below the beam loading limit to reduce beamstrahlung effects greatly reduces the efficiency of the LPA. In the beamstrahlung-limited regime $\left(N_{\text {beam }}<N_{\mathrm{BL}}\right)$, for a single bunch, the efficiency scales as $\eta_{p b}=N_{\text {beam }} W_{\text {stage }} / U_{L} \propto$ $n^{3 / 4}$. Column I in Table III shows the basic plasma density scalings in this beamstrahlung-limited regime, assuming single-bunch operation and $\sigma_{z} \propto \lambda_{p}$. The poor efficiency scaling results in the required $\mathrm{AC}$ power increasing dramatically with decreasing plasma density $P_{\text {wall }} \propto 1 / n$ (assuming constant laser efficiency, as discussed in Sec. III B).

In addition to reducing the bunch charge, beamstrahlung effects may also be mitigated by using shorter beams. Using shorter bunches is limited by bunch density constraints to avoid the blow-out regime [13,14], but as the bunch charge is reduced below the beam loading limit, the bunch length may be reduced proportionally such that $N / \sigma_{z}=$ constant. Injecting proportionally shorter bunches into the LPA will allow more charge per bunch for fixed beamstrahlung, and therefore improved efficiency. If the bunch length is reduced proportionally to the bunch charge (i.e., fixed ratio of beam density to plasma density $n_{b} / n_{0}$ ), Eq. (8) indicates that the bunch charge is independent of the plasma density, and, hence, the efficiency of energy transfer between the plasma wave and beam scales as $\eta_{p b}=N_{\text {beam }} W_{\text {stage }} / U_{L} \propto n^{1 / 2}$. Again, this indicates the advantage of using higher plasma densities. Also note that $Y$ is fixed for $N / \sigma_{z}=$ constant [cf. Eq. (2)]. Column II in Table III shows the basic plasma density scalings in the beamstrahlung-limited regime, assuming single-bunch operation and fixed $n_{b} / n_{0}$. The efficiency scaling $\eta_{p b} \propto n^{1 / 2}$ results in the required AC power scaling $P_{\text {wall }} \propto n^{-1 / 2}$ (assuming constant laser efficiency). The efficiency scalings, and, hence, total power requirements shown in columns I and II of Table III prohibit operating at low densities in the beamstrahlung-limited regime using a single bunch, and indicate the need to consider multibunch trains.

The plasma to beam efficiency can be greatly improved in the beamstrahlung-limited regime $\left(N=N_{\text {beam }}<N_{\text {BL }}\right)$

TABLE III. Plasma density and laser wavelength scalings in the beamstrahlung-limited $\left(N=N_{\text {beam }}<N_{\mathrm{BL}}\right)$ regime: (I) single-bunch operation with $\sigma_{z} \propto \lambda_{p}$, (II) single-bunch operation with $n_{b} / n_{0}$ fixed, (III) multibunch operation with $\sigma_{z} \propto$ $\lambda_{p}$, (IV) multibunch operation with $n_{b} / n_{0}$ fixed.

\begin{tabular}{lcccc}
\hline \hline Parameter & I & II & III & IV \\
\hline Bunch charge, $N_{\text {beam }}$ & $n^{1 / 4}$ & 1 & $n^{1 / 4}$ & 1 \\
Bunch duration, $\sigma_{z}$ & $n^{-1 / 2}$ & 1 & $n^{-1 / 2}$ & 1 \\
Bunch number, $M_{b}$ & $\ldots$ & $\ldots$ & $n^{-3 / 4}$ & $n^{-1 / 2}$ \\
Laser repetition rate, $f_{L}$ & $n^{-1 / 2}$ & 1 & $n^{1 / 4}$ & $n^{1 / 2}$ \\
Average power, $P_{\text {avg }}$ & $n^{-2} \lambda^{-2}$ & $n^{-3 / 2} \lambda^{-2}$ & $n^{-5 / 4} \lambda^{-2}$ & $n^{-1} \lambda^{-2}$ \\
Efficiency, $\eta_{p b}$ & $n^{3 / 4}$ & $n^{1 / 2}$ & 1 & 1 \\
Total AC power, $P_{\text {wall }}$ & $n^{-1}$ & $n^{-1 / 2}$ & $n^{-1 / 4}$ & 1 \\
\hline \hline
\end{tabular}


by using multiple bunches behind the laser. If one considers multiple bunches loaded in the plasma wave such that the total charge in the multibunch train is equal to the beam loading limit, with $M_{b}=N_{\mathrm{BL}} / N_{\text {beam }}$ the number of bunches, then the maximum LPA efficiency can be recovered and the beam to plasma efficiency will then be independent of plasma density. Here we consider multiple bunches loaded behind a single drive laser in the beamstrahlung-limited regime. Note that the ability to use tapered plasmas will be severely limited with multibunch operation (resulting in reduced laser to plasma wave efficiency).

In the beamstrahlung-limited regime, assuming multibunch operation, with fixed IP focusing and luminosity goal, requires the laser repetition rate

$$
f_{L}=\frac{4 \pi \sigma_{x}^{*} \sigma_{y}^{*} \mathcal{L}}{M_{b} N_{\text {beam }}^{2}}=\frac{4 \pi \sigma_{x}^{*} \sigma_{y}^{*} \mathcal{L}}{N_{\mathrm{BL}} N_{\text {beam }}},
$$

and the average laser power per stage required is $P_{\text {avg }}=$ $f_{L} U_{L}$. Note that Eq. (12) assumes that a bunch in the multibunch beam will only interact with a single bunch of the counterpropagating beam at the IP. A single bunch may interact with more than one counterpropagating bunch if the final focus beta function is larger than the bunch spacing $\beta_{*} \gg \lambda_{p}$. Here we are considering typical parameters such that $\beta_{*} \lesssim \lambda_{p}$ (e.g., $\lambda_{p} \sim$ $0.2-1 \mathrm{~mm}$ ), where we do not expect a single bunch to interact strongly with multiple counterpropagating bunches at the IP. Using Eq. (12), the total power required scales as $P_{\text {wall }} \propto n^{-1 / 4}$ for $\sigma_{z} \propto \lambda_{p}$ and using multiple bunch trains in the beamstrahlung-limited regime. Assuming fixed $n_{b} / n_{0}$ the total power requirements are independent of plasma density and laser wavelength using multiple bunch trains in the beamstrahlung-limited regime. The basic plasma density and laser wavelength scalings in the beamstrahlung-limited $\left(N_{\text {beam }}<N_{\mathrm{BL}}\right)$ regime using multibunch trains are shown in Table III, columns III (with $\sigma_{z} \propto \lambda_{p}$ ) and IV (with constant $n_{b} / n_{0}$ ).

\section{B. Collider power requirements}

It is critical to reduce the operating costs of any future collider, and one option for reducing the cost is to reduce the required $\mathrm{AC}$ power for the collider. The total $\mathrm{AC}$ power required to power the linacs is

$$
P_{\text {wall }}=2 N_{\text {stage }} P_{\text {avg }} / \eta_{w L}=\left(2 N_{\text {stage }} U_{L} / \eta_{w L}\right) f_{L},
$$

where $N_{\text {stage }}$ is the number of stages in one linac, $P_{\text {avg }}=$ $f_{L} U_{L}$ is the average laser power per stage, and $\eta_{w L}$ is the efficiency of energy transfer from the wall to the laser. The total efficiency from wall to beam is $\eta=\eta_{w L} \eta_{L p} \eta_{p b}$, with $\eta_{L p}$ the efficiency of energy transfer from the laser to the plasma wave, and $\eta_{p b}$ is the efficiency of energy transfer from the plasma wave to the beam (potentially consisting of $M_{b}$ multiple bunches). Note that LPA-based linacs use plasma wakefields to provide focusing, alleviating the need for conventional external focusing magnets, thereby achieving some power savings. The efficiency of energy transfer from the laser to the plasma $\eta_{L p}$ will remain constant as one scales density with fixed $a_{0}, k_{p} c \tau_{L}$, and $k_{p} r_{L}$. If we assume that in the beamstrahlung-limited regime one can operate with multiple bunches to the beam loading limit $M_{b} N_{\text {beam }}=N_{\mathrm{BL}}$, then the efficiency of energy transfer from plasma wave to beam $\eta_{p b}$ is also independent of density.

For plasma densities sufficiently large that the charge is not limited by beamstrahlung considerations (i.e., $N_{\text {beam }} \geq N_{\mathrm{BL}}$ ) it was originally shown in Ref. [6] that the total power scaled as $P_{\text {wall }} \propto n^{1 / 2}$. This indicates that, if total $\mathrm{AC}$ power is to be minimized for single-bunch operation with the constraint of fixed acceptable beamstrahlung, then the optimal plasma density is where $N_{\text {beam }} \simeq N_{\mathrm{BL}}$. Also when employing multibunch trains, it is clearly advantageous to operate at the density where $N_{\text {beam }} \simeq N_{\mathrm{BL}}$, since there is no reduction in total power cost by operating at lower plasma density, and there are the disadvantages of lower accelerating gradients $E_{z} \propto n^{1 / 2}$ and larger laser systems $U_{L} \propto n^{-3 / 2}$.

Figure 1 shows an example of the total AC power requirements versus plasma density for a $1 \mathrm{TeV}$ center-of-mass collider with $\sigma_{*}^{2}=100 \mathrm{~nm}^{2}, \mathcal{L}=2 \times 10^{34} \mathrm{~s}^{-2} \mathrm{~cm}^{-2}$, and $\eta=6 \%$. In the beamstrahlung-limited regime both single bunch (solid curves) and multibunch (dashed curves) operation (with fixed $n_{b} / n_{0}$ ) are shown in Fig. 1. Here the total efficiency assumed $\eta_{w L}=30 \%, \eta_{L p}=50 \%$, and $\eta_{p b}=$ $40 \%$. The total AC power required versus plasma density for the constraints $n_{\gamma}=1, n_{\gamma}=2$, and $n_{\gamma}=3$ was considered in Fig. 1. Using Eq. (7), this corresponds to beamstrahlung-induced average fractional energy loss of $\delta_{b} \simeq 28 \%, \delta_{b} \simeq 57 \%$, and $\delta_{b} \simeq 86 \%$, respectively. As indicated by Fig. 1, for these parameters, the total wall-plug

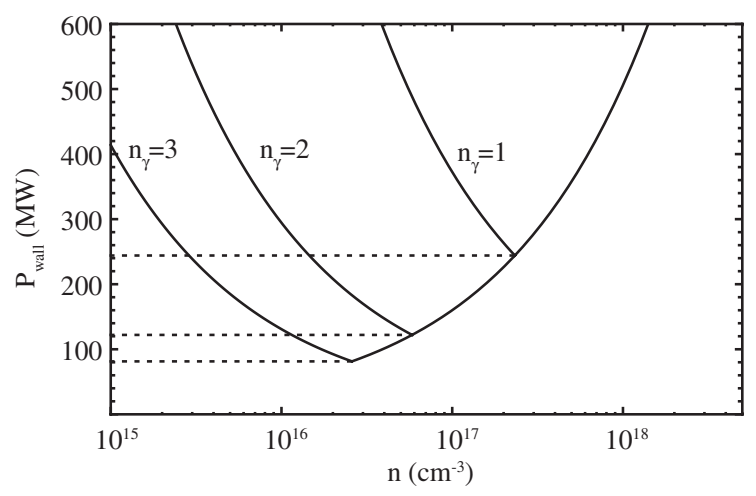

FIG. 1. Total AC power required versus plasma density for a $1 \mathrm{TeV}$ center-of-mass collider with $\sigma_{*}^{2}=100 \mathrm{~nm}^{2}, \mathcal{L}=2 \times$ $10^{34} \mathrm{~s}^{-2} \mathrm{~cm}^{-2}$, and $\eta=6 \%$, with the number of beamstrahlung photons produced per electron bounded with $n_{\gamma}=1, n_{\gamma}=2$, and $n_{\gamma}=3$. The solid line is single-bunch operation and the dashed line is using multibunch trains (with fixed $n_{b} / n_{0}$ ). 
power is minimized for plasma densities of a few $10^{16} \mathrm{~cm}^{-3}$ to a few $10^{17} \mathrm{~cm}^{-3}$ for single-bunch operation, depending on the beamstrahlung that can be tolerated at the IP.

It should be noted that Fig. 1 assumed $\sigma_{*}^{2}=100 \mathrm{~nm}^{2}$ and $\eta=6 \%$. If, for example, the injector for such a collider is able to produce lower emittance beams such that $\sigma_{*}^{2}=25 \mathrm{~nm}^{2}$ is achievable, then, as Eq. (9) indicates, for fixed beamstrahlung, the power requirements shown in Fig. 1 would be reduced by half. Also, if laser technology enables greater efficiencies such that $\eta_{w L}>30 \%$, then the power requirements shown in Fig. 1 would be reduced proportionally.

Figure 1 assumed a constant laser efficiency $\eta_{w L}$ for every operating plasma density. In general, operating at different plasma densities requires different laser characteristics (e.g., laser pulse duration $\tau_{L} \propto n^{-1 / 2}$, laser energy $U_{L} \propto n^{-3 / 2}$, etc.) that may require different laser technologies with varying efficiency. For example, fiber lasers (coherently combined) are considered to be a candidate laser technology for high-peak and high-average laser power generation [21]. Using fiber lasers [22], higher efficiencies $(\sim 30 \%)$ are achieved near $\mathrm{cw}$ operation, while the efficiency drops significantly at lower $(\lesssim 10 \mathrm{kHz})$ repetition rates. At high laser pulse repetition rates $(>10 \mathrm{kHz})$, the rate is sufficiently fast compared to the upper-state lifetime of the rare-earth ions in the glass fiber so that little power is lost to amplified spontaneous emission (ASE) between pulses. However, as the repetition rate is reduced progressively below $10 \mathrm{kHz}$, ASE losses reduce the laser system efficiency. This is, in general, true of any solid-state laser system. Hence, if solid-state laser technology is employed, then higher laser efficiencies are achieved by using higher repetition rates, i.e., LPA operation at higher plasma density.

Table IV shows three illustrative examples of collider parameters with beamstrahlung fixed at $n_{\gamma} \leq 2$. The high density $\left(n=10^{17} \mathrm{~cm}^{-3}\right)$ option shown in Table IV would use multiple $\left(N_{\text {stage }}=50\right)$ LPA stages to reach $U_{b}=$ $0.5 \mathrm{TeV}$, and the bunch charge would not be beamstrahlung limited $\left(N_{\text {beam }}>N_{\mathrm{BL}}=N\right.$ with $\left.n_{\gamma}=1.5\right)$. In this example, operating at $n=10^{17} \mathrm{~cm}^{-3}$ would require $32 \mathrm{~J}$ of laser energy and $480 \mathrm{~kW}$ of average laser power. Table IV also shows a low density $\left(n=2 \times 10^{15} \mathrm{~cm}^{-3}\right)$ option that uses a single LPA stage to reach $U_{b}=0.5 \mathrm{TeV}$, in the beamstrahlung-limited regime $\left(N_{\mathrm{BL}}>N_{\text {beam }}=N\right)$. Both single-bunch $\left(M_{b}=1\right)$ and multibunch train $\left(M_{b}=5\right)$ operation are considered.

The stage length listed in Table IV comprises the plasma length and the laser coupling distance from final optic to the plasma (see Ref. [6] for a discussion on optimization of the stage length). The plasma considered here consists of a preformed plasma channel for optically guiding the short-pulse laser [3]. The required laser coupling distance to the final optic increases with decreasing density; using conventional optics, to avoid damage on the optic
TABLE IV. Three example parameter sets for a $1 \mathrm{TeV}$ laserplasma linear collider operating at plasma densities $n_{0}=$ $10^{17} \mathrm{~cm}^{-3}$ and $2 \times 10^{15} \mathrm{~cm}^{-3}$ with the beamstrahlung constraint $n_{\gamma} \leq 2$.

\begin{tabular}{lccc}
\hline \hline Plasma density, $n_{0}\left[10^{16} \mathrm{~cm}^{-3}\right]$ & 10 & 0.2 & 0.2 \\
\hline Plasma wavelength, $\lambda_{p}[\mathrm{~mm}]$ & 0.1 & 0.75 & 0.75 \\
Energy, center of mass, $U_{\mathrm{cm}}[\mathrm{TeV}]$ & 1 & 1 & 1 \\
Beam energy, $\gamma m c^{2}=U_{b}[\mathrm{TeV}]$ & 0.5 & 0.5 & 0.5 \\
Luminosity, $\mathcal{L}\left[10^{34} \mathrm{~s}^{-1} \mathrm{~cm}^{-2}\right]$ & 2 & 2 & 2 \\
Number per bunch, $N\left[10^{9}\right]$ & 4 & 5.2 & 5.2 \\
Laser repetition rate, $f_{L}[\mathrm{kHz}]$ & 15 & 8.7 & 1.7 \\
Number of bunches in beam, $M_{b}$ & 1 & 1 & 5 \\
Bunch length, $\sigma_{z}[\mu \mathrm{m}]$ & 1.0 & 1.3 & 1.3 \\
Beam size at IP, $\sigma_{x}^{*}=\sigma_{y}^{*}[\mathrm{~nm}]$ & 10 & 10 & 10 \\
Beamstrahlung parameter, $Y$ & 180 & 180 & 180 \\
Beamstrahlung photons, $n_{\gamma}$ & 1.5 & 2 & 2 \\
Beamstrahlung energy loss, $\delta_{\gamma}$ & 0.42 & 0.55 & 0.55 \\
Disruption parameter, $D$ & 0.12 & 0.20 & 0.20 \\
Energy gain/stage, $U_{\text {stage }}[\mathrm{GeV}]$ & 10 & 500 & 500 \\
Stage length ${ }^{\mathrm{a}}, L_{\text {stage }}[\mathrm{m}]$ & 2 & 500 & 500 \\
Laser energy per stage, $U_{L}[\mathrm{~kJ}]$ & 0.032 & 11 & 11 \\
Laser wavelength, $\lambda[\mu \mathrm{m}]$ & 1 & 1 & 1 \\
Laser pulse duration, $\tau_{L}[\mathrm{fs}]$ & 56 & 396 & 396 \\
Initial normalized laser intensity, $a_{0}$ & 1.5 & 1.5 & 1.5 \\
Average laser power/stage $P_{\text {avg }}[\mathrm{MW}]$ & 0.48 & 98 & 19 \\
Number of stages $(1 \mathrm{linac}), N_{\text {stage }}$ & 50 & 1 & 1 \\
Linac length $\left(1\right.$ beam), $L_{\text {total }}[\mathrm{km}]$ & 0.1 & 0.5 & 0.5 \\
Efficiency (wall plug to beam) $[\%]$ & 6 & 1 & 6 \\
Total wall-plug power, $P_{\text {wall }}[\mathrm{GW}]$ & 0.16 & 0.66 & 0.13 \\
\hline \hline & & & \\
\hline
\end{tabular}

${ }^{a}$ LPA length and laser in-coupling distance.

(e.g., a fluence $F \lesssim 1 \mathrm{~J} / \mathrm{cm}^{-2}$ for sub-ps pulses), the required laser coupling distance is hundreds of Rayleigh ranges, $L \gtrsim Z_{R}\left[I /\left(F \omega_{p}\right)\right]^{1 / 2} \propto 1 /\left(n^{5 / 4} \lambda\right)$. Novel optical techniques, such as the use of plasma mirrors [23], are actively being researched.

Note that the illustrative examples shown in Table IV are not optimized. For example, since the total power required is independent of density with multibunch trains, it is more advantageous to operate at higher density (with higher accelerating gradients, smaller lasers, etc.) with the same total power requirements. As shown in Fig. 1, operating at $n=5.8 \times 10^{16} \mathrm{~cm}^{-3}$ achieves the same collider parameters (luminosity, center-of-mass energy, and beamstrahlung at the IP $n_{\gamma}=2$ ) with the same total power required as operating at $n=2 \times 10^{15} \mathrm{~cm}^{-3}$ (cf. dashed curve in Fig. 1), while achieving a higher accelerating gradient and using smaller laser systems.

\section{SUMMARY AND CONCLUSIONS}

In this work we have considered the influence of IP beam-beam interaction constraints on the basic plasma density scalings for a linear collider based on laser-plasma accelerators. The maximum charge in a single bunch is 
limited either by beam loading or by beamstrahlung. At high plasma densities, the single-bunch charge is limited by beam loading and the required $\mathrm{AC}$ power scales as $P_{\text {wall }} \propto n^{1 / 2}$, as was previously discussed in Ref. [6]. Operating at low plasma density increases beamstrahlung effects $n_{\gamma} \simeq 0.35 \delta_{b} \propto n^{-1 / 2}$ for $Y \gg 1$, owing to the high bunch charge and longer bunch length at lower plasma density. Beamstrahlung can be constrained for fixed luminosity, at the cost of using smaller charge per bunch at higher laser repetition rate and, hence, higher power requirements. If the bunch charge is limited by beamstrahlung, then the required AC power scales as $P_{\text {wall }} \propto n^{-1 / 2}$ using single bunches.

Multibunch operation may be considered for improved efficiency in the beamstrahlung-limited regime. If the collider is constrained by beamstrahlung then the required $\mathrm{AC}$ power is independent of plasma density using multiple bunch trains. Since the AC power is independent of plasma density using multibunch trains in the beamstrahlunglimited regime, there are significant advantages to operating at higher density where $N_{\text {beam }} \simeq N_{\mathrm{BL}}$ and the required beam energy and luminosity can be achieved, within the constrains of beamstrahlung, at the same total power cost, but with higher accelerating gradients and smaller laser systems.

In this work we considered round beams. Flat beams may also be employed in LPAs to reduce beamstrahlung, but using flat beams requires the use of highly asymmetric laser modes for matched beam propagation, as well as LPA injectors compatible with damping rings for asymmetric emittance generation and significantly more challenging alignment tolerances at the IP.

Although power and IP considerations are critical, we expect that the plasma density choice, as well as other parameter choices, will be dictated by the available laser technology that develops for efficient, high-peak, and average power lasers. For example, fiber laser technology efficiency favors high laser repetition rates, and, therefore, LPA operation at high plasma densities.

A TeV linear collider is extremely challenging for any accelerator technology. Although LPA technology has made rapid experimental progress in recent years, significant laser technology developments are required, as well as LPA maturity, before a detailed LPA-based collider design (i.e., integrated injector, cooling, LPA-based linac, and final focus) is possible. We anticipate that the LPA-collider design will evolve with better understanding of the laserplasma physics, based on future experimental results, and as the laser technology advances.

\section{ACKNOWLEDGMENTS}

The authors are grateful to C. Benedetti, C. Geddes, Cs. Tóth, and J. van Tilborg for many useful discussions. The authors also acknowledge stimulating discussions with participants at the Second Joint ICFA-ICUIL Workshop for High Power Laser Technology for Future Accelerators, and, in particular, with R. Assmann, M. Battaglia, W. Chou, J. Dawson, and K. Yokoya. This work was supported by the Director, Office of Science, Office of High Energy Physics, of the U.S. Department of Energy under Contract No. DE-AC02-05CH11231.

\section{APPENDIX: BEAM-BEAM COHERENT PAIR PRODUCTION}

For high-energy colliders $(\geq 1 \mathrm{TeV})$ there is a significant probability of coherent pair production during the beam-beam collision, which can be an important source of detector background [24]. In the limit $Y \gg 1$, the average number of electron-positron pairs created per primary electron is [10]

$$
\begin{aligned}
n_{e^{+} e^{-}} & \simeq 0.295\left(\frac{\alpha^{2} \sigma_{z}}{r_{e} \gamma}\right)^{2}(\ln \Upsilon-2.488) \Upsilon^{4 / 3} \\
& \simeq 0.0457(\ln \Upsilon-2.488) n_{\gamma}^{2} .
\end{aligned}
$$

If the number of beamstrahlung photons $n_{\gamma}$ is held fixed, and $n_{b} / n_{0}$ is fixed, then the number of coherent pairs $n_{e^{+}} e^{-}$ will be constant in the $Y \gg 1$ regime. Hence, constraining the beamstrahlung photon emission $n_{\gamma}$ as described in Sec. III, also constrains the coherent pair production. For the $n=10^{17} \mathrm{~cm}^{-3}$ example in Table IV, $n_{e^{+}} e^{-} \approx 0.29$.

[1] I. Hinchliffe and M. Battaglia, Phys. Today 57, 49 (2004).

[2] G. Dugan, in Advanced Accelerator Concepts, edited by V. Yakimenko (AIP, New York, 2004), Vol. 737, pp. 29-60.

[3] E. Esarey, C. B. Schroeder, and W. P. Leemans, Rev. Mod. Phys. 81, 1229 (2009).

[4] W. P. Leemans, B. Nagler, A. J. Gonsalves, C. Tóth, K. Nakamura, C. G. R. Geddes, E. Esarey, C. B. Schroeder, and S. M. Hooker, Nature Phys. 2, 696 (2006).

[5] W. Leemans and E. Esarey, Phys. Today 62, 44 (2009).

[6] C. B. Schroeder, E. Esarey, C. G. R. Geddes, C. Benedetti, and W. P. Leemans, Phys. Rev. ST Accel. Beams 13, 101301 (2010).

[7] K. Nakajima, A. Deng, X. Zhang, B. Shen, J. Liu, R. Li, Z. Xu, T. Ostermayr, S. Petrovics, C. Klier, K. Iqbal, H. Ruhl, and T. Tajima, Phys. Rev. ST Accel. Beams 14, 091301 (2011).

[8] P. Chen and K. Yokoya, Phys. Rev. D 38, 987 (1988).

[9] T. Himel and J. Siegrist, AIP Conf. Proc. 130, 602 (1985); R. Blankenbecler and S. D. Drell, Phys. Rev. D 36, 277 (1987).

[10] P. Chen, in Handbook of Accelerator Physics and Engineering, edited by A.W. Chao and M. Tigner (World Scientific, Singapore, 1999) pp. 140-144; K. Yokoya and P. Chen, in Frontiers of Particle Beams: Intensity Limitations, Lecture Notes in Physics Vol. 400, edited by M. Dienes, M. Month, and S. Turner (SpringerVerlag, Berlin, 1992), pp. 415-445. 
[11] CLIC Conceptual Design Report, Technical Report, CERN, 2011.

[12] J. Delahaye, G. Guignard, T. Raubenheimer, and I. Wilson, Nucl. Instrum. Methods Phys. Res., Sect. A 421, 369 (1999).

[13] J. B. Rosenzweig, B. Breizman, T. Katsouleas, and J. J. Su, Phys. Rev. A 44, R6189 (1991).

[14] J. B. Rosenzweig, A. M. Cook, A. Scott, M. C. Thompson, and R. B. Yoder, Phys. Rev. Lett. 95, 195002 (2005).

[15] T. Katsouleas, S. Wilks, P. Chen, J. M. Dawson, and J. J. Su, Part. Accel. 22, 81 (1987).

[16] K. Oide, Phys. Rev. Lett. 61, 1713 (1988).

[17] W. Rittershofer, C. B. Schroeder, E. Esarey, F. J. Grüner, and W.P. Leemans, Phys. Plasmas 17, 063104 (2010).
[18] C. B. Schroeder, C. Benedetti, E. Esarey, and W.P. Leemans, Phys. Rev. Lett. 106, 135002 (2011).

[19] E. Cormier-Michel, E. Esarey, C. G. R. Geddes, C. B. Schroeder, K. Paul, J. P. Mullowney, J. R. Cary, and W. P. Leemans, Phys. Rev. ST Accel. Beams 14, 031303 (2011).

[20] R. Assmann and K. Yokoya, Nucl. Instrum. Methods Phys. Res., Sect. A 410, 544 (1998).

[21] G. A. Mourou, D. Hulin, and A. Galvanauskas, AIP Conf. Proc. 827, 152 (2006).

[22] D. J. Richardson, J. Nilsson, and W. A. Clarkson, J. Opt. Soc. Am. B 27, B63 (2010).

[23] D. Panasenko, A. J. Shu, A. Gonsalves, K. Nakamura, N. H. Matlis, C. Toth, and W. P. Leemans, J. Appl. Phys. 108, 044913 (2010).

[24] P. Chen and V. I. Telnov, Phys. Rev. Lett. 63, 1796 (1989). 